\section{Optimal Maintenance, Repairmen, and Control Strategies for Systems with Breakdowns}

\author{
V.V.S. SARMA AND MANSOOR ALAM
}

Abstract-Optimal preventive maintenance and repairmen policies for a system of machines subject to degradation with age and intermittent breakdowns and repairs are derived using optimal control theory. When this system of machines forms part of a dynamic process control system, a method of obtaining optimal closed-loop control law is indicated.

\section{INTRODUCTION}

Many large industrial systems have two distinct kinds of problems, the problems of management and the problems of operation. The former are mostly economic decision problems such as resource allocation, maintenance, repair and replacement of equipment, personnel, etc. The second type deals with efficient technical operation of the processes and is in the domain of automatic control. It is being increasingly realized that both these types of problems are amenable for solution via control theory and mathematical programming. There are also interactions between the two kinds of problems which need coordination at both levels for efficient operation of the integrated system. As an example, we might consider the management and operation of a large interconnected power system. The maintenance scheduling of generators, replacement of older machines, and man power planning form part of the economic decision aspect while the technical operation of the system involves maintaining appropriate voltage levels at various points; regulating the active and reactive power flows while maintaining the dynamic stability of the system in the face of machine shut down due to maintenance or breakdowns.

The problem of optimal maintenance of equipment has been the subject of considerable research [1], [2]. Thompson [3] considered a machine's degradation with age and obtained optimal preventive maintenance (PM) policy via Pontryagin's maximum principle. His results were subsequently generalized in several recent papers [4H[8]. Sworder and Kazangey [9] modeled a production process with several machines breaking down at random points in time as an LQG problem with jump parameters and derived an optimal feedback controller.

In this short paper, we consider a generalization of Thompson's model [3] for a system of machines taking into account intermittent breakdowns and repairs to obtain optimal PM and repairmen policies. Then we examine the implications of these in a process control situation in which this system of machines forms a part, using Sworder's approach [9].

\section{Optimal Maintenance and Repairmen POLICIES FOR A NUMBER OF MACHINES}

In this section, the various models are described in a form amenable for solution via optimal control theory.

\section{A. Model for Machine Deterioration}

Following Thompson [3], the model for the gradual deterioration of a system of machines with age, which can be partly offset via PM, is given by

$$
d S(t) / d t=-d(t)+f(t) u(t)
$$

In (1), $S(t)$ is the salvage value of the system of machines in dollars, $d(t)$ is the inferiority gradient, $f(t)$ is the maintenance effectiveness function, and $u(t),(0 \leqslant u(t) \leqslant U)$, is the number of dollars spent on PM, over and above necessary repairs.

Manuscript received December 11, 1974; revised November 13, 1975. Paper recommended by B. Friedland, Chairman of the IEEE S-CS Applications, Systems Evaluation, Components Committee.

V.V.S. Sarma is with the Department of Electrical Communication Engineering, Indian V.V.S. Sarma is with the Department
Institute of Science, Bangalore, India.

M. Alam was with the School of Automation, Indian Institute of Science, Bangalore, India. He is now with the Department of Electrical Engineering and Electronics, University of Liverpool, Liverpool, England.
Remark 1: It may, however, be noted that the simple linear model (1) may not completely represent the observed economic behavior. In general, an equation such as

$$
d S(t) / d t=-d(t)+g(S(t), u(t))
$$

may be more appropriate. Usually $g(\cdots)$ satisfies conditions such as 1$)$ $g(S, 0)=0$, implying that $S(t)$ satisfies the linear relation when PM is zero and 2) $g_{u}(S, u)>0$, indicating that a positive maintenance is always beneficial. Conditions on $g_{S}(S, u)$ and $g_{u u}(S, u)$ may also be used if PM is not uniformly effective at all conditions of the machine and at all times. The simpler model (1) satisfies conditions 1) and 2). Luenberger [10] recently considered models analogous to (2). Arora and Lele's model [4] is a special case of (1) and (2) [6].

Remark 2: Extension of Thompson's model for a single machine to a system of machines involves the assumption that all machines are bought at the same time. If they are bought at different times, equations of the form (1) may be written for separate machines to determine individual PM policies. Otherwise, a policy of the form $u(t)=1$ for all machines gives rise to the problem of maintenance allocation.

\section{B. Model for Intermittent Breakdowns and Repairs}

We assume that the machines normally require no human care and that there are $N$ machines and $m(m \leqslant N)$ repairmen in the system. At any time $t$, a machine may fail and call for service. Repair work is initiated immediately after breakdown if a repairman is free or else a broken down machine joins a queue. The system of machines, at time $t$, is characterized by state $i$, where $i=0,1,2, \cdots, N$ denotes the number of inoperative machines at $t$. If $\lambda$ and $\mu$ characterize the breakdown and repair processes of each machine assumed exponential, the equations governing the probabilities $P_{i}^{m}(t)$ of finding the system in state $i$ are given by [11]

$$
\begin{aligned}
d P_{0}^{m}(t) / d t= & -N \lambda P_{0}^{m}(t)+\mu P_{1}^{m}(t) \\
d P_{i}^{m}(t) / d t= & -\{(N-i) \lambda+i \mu\} P_{i}^{m}(t)+(N-i+1) \lambda P_{i-1}^{m}(t) \\
& +(i+1) \mu P_{i+1}^{m}(t), \quad 1 \leqslant i \leqslant m-1 \\
d P_{j}^{m}(t) / d t= & -\{(N-j) \lambda+m \mu\} P_{j}^{m}(t)+(N-j+1) \lambda P_{j-1}^{m}(t) \\
& +m \mu P_{j+1}^{m}(t), \quad m \leqslant j \leqslant N-1 \\
d P_{N}^{m}(t) / d t= & -m \mu P_{N}^{m}(t)+\lambda P_{N-1}^{m}(t) .
\end{aligned}
$$

The breakdown rate $\lambda$ is usually reduced by PM as shown by

$$
\lambda=\lambda_{0}-\lambda_{1} u
$$

where $\lambda_{0}$ is the breakdown rate without PM and $\lambda_{1}$ denotes the effectiveness of PM in bringing down the breakdown rate. In practice, $\lambda_{1} \ll \lambda_{0}$ such that $\lambda$ is always positive, thus implying that PM cannot prevent breakdowns.

\section{Performance Index}

The objective is to choose the optimal PM policy $u^{*}$, the optimum number of repairmen $m^{*}$ and the sale date $T$ to maximize the expected profit from the given system of machines. The performance index $V(T)$ denotes discounted profit during the life of the system of machines plus the discounted solvage value at time $T$

$$
\begin{aligned}
E\{V(T)\}=S(T) e^{-r T} & \\
+ & \int_{0}^{T}\left\{p S(t)\left[P_{0}^{m}(t)+\alpha_{1} P_{1}^{m}(t)+\cdots+\alpha_{N-1} P_{N-1}^{m}(t)\right]\right. \\
& \quad-\gamma m(t)-u(t)-\left[k_{1} P_{1}^{m}(t)+k_{2} P_{2}^{m}(t)\right. \\
& \left.\left.+\cdots+k_{N} P_{N}^{m}(t)\right]\right\} e^{-r t} d t .
\end{aligned}
$$

In (5) $p$ is the production rate at $t$, defined as output value at $t /$ salvage value $t, r$ is the rate of interest, $T$ is the sale date, and $\gamma$ is the labor rate per unit time. The parameters $\alpha$ and $k\left(0<\alpha_{N-1}<\alpha_{N-2}<\cdots \alpha_{2}<\alpha_{1}\right.$ $<1)$ and $\left(k_{1}<k_{2}<\cdots<k_{N}\right)$ characterize the decreased productivity and the increase in additional repair expenditure as the number of 
inoperative machines increases. It is assumed that the output of the machine is obtained only when the machines are working and that the down time, because of PM and repairs, is negligible compared to the total planning horizon $T$.

The state equations (1) and (3) and the performance index (5) are now in a form amenable for solution via the maximum principle [12]. The number of repairmen $m$ may be treated as a constant throughout the planning horizon and $N$ optimal control problems may be solved with $m=1,2, \cdots, N$ to obtain the optimal number of repairmen. Otherwise $m(t)$ may be assumed a control function and solved to obtain the optimal repairmen policy along with optimal policy. Several examples are considered in Section III. Numerical solutions are obtained by using the conjugate gradient algorithm developed by Pagurek and Woodside [13] for optimal control problems with bounded control variables. Convergence to optimum is obtained in a small number of iterations and the details are given in [14].

\section{Examples}

Example 1: (A single machine case.) We consider Thompson's example [3] with intermittent breakdowns and repairs. $S(0)=100, d(t)=2$, $p=0.1, r=0.05, k_{1}=1, \gamma=0.5, U=1, f(t)=2 /(1+t)^{\frac{1}{2}}, \lambda_{0}=0.05, \lambda_{1}=$ 0.005 , and $\mu=0.5$. Thompson's policy (without breakdowns) is as follows:

$$
\begin{aligned}
u^{*}(t) & =1, & & 0 \leqslant t \leqslant 10.6 \\
& =0, & & 10.6<t \leqslant 34.8
\end{aligned}
$$

sale date $=34.8$.

Optimal PM policy with breakdowns and repairs is as follows:

$$
\begin{aligned}
u^{*}(t) & =1, & & 0 \leqslant t \leqslant 6.7 \\
& =0, & & 6.7<t \leqslant 26.2
\end{aligned}
$$

sale date $=26.2$.

Example 2: (A two machine case-number of repairmen constant throughout the planning horizon.) For the two machine problem without PM dependent breakdown rate, the probability equations (3) may be solved analytically.

1) $N=2, m=1$, for $P_{0}^{1}(0)=1, P_{1}^{1}(0)=0$, and $P_{2}^{1}(0)=0$.

$P_{0}^{\prime}(t)=\frac{1}{1+\rho+\rho^{2}}+\frac{\rho}{2}\left[\frac{e^{-(\lambda+\mu) t+t \sqrt{\lambda \mu}}}{1+\rho-\sqrt{\rho}}+\frac{e^{-(\lambda+\mu) t-i \sqrt{\lambda \mu}}}{1+\rho+\sqrt{\rho}}\right]$

$P_{1}^{\prime}(t)=\frac{\rho}{1+\rho+\rho^{2}}$

$$
-\frac{\rho}{2}\left[\frac{e^{-(\lambda+\mu) t+t \sqrt{\lambda \mu}}}{1+\rho-\sqrt{\rho}}(1-\sqrt{\rho})+\frac{e^{-(\lambda+\mu) t-r \sqrt{\lambda \mu}}}{1+\rho+\sqrt{\rho}}(1+\sqrt{\rho})\right]
$$

$P_{2}^{\prime}(t)=\frac{\rho^{2}}{1+\rho+\rho^{2}}+\frac{\rho \sqrt{\rho}}{2}\left[-\frac{e^{-(\lambda+\mu)_{t}+\mathrm{r} \sqrt{\lambda \mu}}}{1+\rho-\sqrt{\rho}}+\frac{e^{-(\lambda+\mu) t-t \sqrt{\lambda \mu}}}{1+\rho+\sqrt{\rho}}\right]$

where $p=\lambda / \mu$.

The optimal PM policy for this case is given by $u^{*}(t)=U$ if

$$
\begin{array}{r}
f(t)>e^{-r t} /\left[p \left\{e^{-r T}\left(1 / P+A+B e^{-(\lambda+\mu) T+T \sqrt{\lambda \mu}}+C e^{-(\lambda+\mu) T-T-\sqrt{\lambda \mu}}\right)\right.\right. \\
\left.\left.-e^{-r t}\left(A+B e^{-(\lambda+\mu) t+r \sqrt{\lambda \mu}}+C e^{-(\lambda+\mu) t-\lambda \sqrt{\lambda \mu}}\right)\right\}\right]
\end{array}
$$

where

$$
\begin{aligned}
A & =-\frac{1+\rho / 2}{\left(1+\rho+\rho^{2}\right) r} \\
B & =\left[\frac{\rho / 2-\rho(1-\sqrt{\rho}) / 4}{1+\rho-\sqrt{\rho}}\right]\left[\frac{1}{-(\lambda+\mu)+\sqrt{\lambda \mu}-r}\right]
\end{aligned}
$$

and

$$
C=\left[\frac{\rho / 2-(1+\sqrt{\rho}) / 4}{1+\rho+\sqrt{\rho}}\right]\left[\frac{1}{-(\lambda+\mu)-\sqrt{\lambda} \mu-r}\right] .
$$

$u^{*}(t)=0$, otherwise

2) $N=2, m=2$, for $P_{0}^{2}(0)=1, P_{1}^{2}(0)=0, P_{2}^{2}(0)=0$.

$$
\begin{aligned}
& P_{0}^{2}(t)=\frac{\mu^{2}}{(\lambda+\mu)^{2}}+\frac{2 \lambda \mu}{(\lambda+\mu)^{2}} e^{-(\lambda+\mu) t}+\frac{\lambda^{2}}{(\lambda+\mu)^{2}} e^{-2(\lambda+\mu) t} \\
& P_{1}^{2}(t)=\frac{2 \lambda \mu}{(\lambda+\mu)^{2}}+\frac{2 \lambda(\lambda-\mu)}{(\lambda+\mu)^{2}} e^{-(\lambda+\mu) t}-\frac{2 \lambda^{2}}{(\lambda+\mu)^{2}} e^{-2(\lambda+\mu) r} \\
& P_{2}^{2}(t)=\frac{\lambda^{2}}{(\lambda+\mu)^{2}}-\frac{2 \lambda^{2}}{(\lambda+\mu)^{2}} e^{-(\lambda+\mu) t}+\frac{\lambda^{2}}{(\lambda+\mu)^{2}} e^{-2(\lambda+\mu) r}
\end{aligned}
$$

The optimal PM policy for this case is given by $u^{*}(t)=U$ if

$$
\begin{aligned}
f(t)>e^{-r} /\left[p \left\{e^{-r T}\left(1 / p+A^{\prime}+B^{\prime} e^{-(\lambda+\mu) T}\right)\right.\right. & \\
& \left.\left.-e^{-r}\left(A^{\prime}+B^{\prime} e^{-(\lambda+\mu) t}\right)\right\}\right]
\end{aligned}
$$

where $A^{\prime}=-\mu /(\lambda+\mu) r, B^{\prime}=-\lambda /(\lambda+\mu)(\lambda+\mu+r)$, and $u^{*}(t)=0$ otherwise.

The following numerical values of various parameters were employed for computation of optimal maintenance policies.

$$
\begin{aligned}
& S(0)=200, d(t)=4, p=0.2, r=0.05, \alpha_{1}=\frac{1}{2}, k_{1}=1, k_{2}=2 \\
& f(t)=4 /(1+t)^{\frac{1}{2}}, \lambda=0.05, \mu=1.0, \gamma=0.5, U=1 .
\end{aligned}
$$

The results are shown in Table I. The optimal number of repairmen $=1$.

Example 3: (A two machine case-optimal repairmen policies.) Here $m(t)$ is treated as a control variable and (5) is maximized subject to (1) and (3) and constraints $0 \leqslant u(t) \leqslant U$ and $1 \leqslant m(t) \leqslant 2$. The numerical values assumed are $S(0)=200, d(t)=4, p=0.2, r=0.05, f(t)=2 /(1+t)^{\frac{1}{2}}$, $\lambda=0.05, \mu=1, U=1, a_{1}=\frac{1}{2}, k_{1}=1$, and $k_{2}=2$. The results are shown in Table II.

\section{Process Control Systey}

While the economic decision aspect of optimizing return from an equipment is almost similar for several applications. the process control aspect depends on a particular application. The breakdowns and repairs of the machines affect the process control indirectly as seen from the power system example and this aspect can be modeled using Sworder's approach [9].

Let us assume that the system of machines considered in Section II forms part of a process control system described by the state equation

$$
d x(t) / d t=A(t) x(t)+B(t) v(t), \quad x(0)=x_{0} .
$$

Matrices $A$ and $B$ depend upon the operational status of the system, i.e., the number of inoperative machines. Breakdowns of machines cause jumps in parameter matrices of the system

$$
\left[A_{0}, B_{0}\right] \rightarrow\left[A_{1}, B_{1}\right] \rightarrow \cdots\left[A_{N}, B_{N}\right]
$$

while repair causes transitions in the opposite direction. If we return to the analogy of power systems considered in the introduction the state variables $\boldsymbol{x}$ in (6) are voltage deviations and power fluctuations and this dynamic process is to be controlled by the control vector $t(t)$ describing the action of voltage regulators and speed control governors. Breakdowns in the system and repairs affect the dynamic performance as shown by (6) and (7). There exists a matrix $Q^{m u}(t)$ which governs the transition between the dynamic modes of the system (6) such that

$$
\begin{aligned}
\operatorname{Pr}\{[A(t+\Delta), B(t+\Delta)] & \left.=\left[A_{j}, B_{j}\right][A(t), B(t)]=\left[A_{i}, B_{i}\right]\right\} \\
& =q_{i j}^{m u}(t) \Delta+0(\Delta), \quad i \neq j \\
& =1+q_{i i}^{m u}(t) \Delta+0(\Delta), \quad i=j ; \quad i, j=0, i, \cdots, N
\end{aligned}
$$


TABLE I

Optimal Maintenance Policies for the Two Machine Case

\begin{tabular}{ccccc}
\hline \hline \multirow{2}{*}{$\begin{array}{c}\text { Number of } \\
\text { repairmen }\end{array}$} & $\begin{array}{c}\text { Parameters of Optimal } \\
\text { Maintenance Policy } u^{*}(t)\end{array}$ & $\begin{array}{c}\text { Optimal Net } \\
\text { Profit } V^{*}(T)\end{array}$ & $\begin{array}{c}\text { Optimal Profit } \\
\text { per Unit Time }\end{array}$ \\
\cline { 2 - 3 }$m$ & $\begin{array}{c}\text { Stop Maintenance } \\
T^{\prime}\end{array}$ & $\begin{array}{c}\text { Sale Date } \\
T\end{array}$ & & \\
\hline 1 & 50.5 & 56.9 & 606.8 & 10.6 \\
2 & 52 & 58.6 & 595.1 & 10.1 \\
\hline
\end{tabular}

TABLE II

Optimal Maintenance and Optimal Repairmen Policies for the Two Machine Case

\begin{tabular}{|c|c|c|c|c|c|c|c|}
\hline \multirow{2}{*}{$\begin{array}{c}\text { Serial } \\
\text { Number }\end{array}$} & \multirow{2}{*}{$\begin{array}{l}\text { Labor } \\
\text { Rate }\end{array}$} & \multicolumn{2}{|c|}{$\begin{array}{l}\text { Parameters of Optimal } \\
\text { Maintenance Policy } u^{*}\end{array}$} & \multirow{2}{*}{$\begin{array}{l}\text { Optimal } \\
\text { Profit } \\
V^{*}(T)\end{array}$} & \multirow{2}{*}{\multicolumn{3}{|c|}{ Optimal Repairmen Policy $m^{*}$}} \\
\hline & & $\begin{array}{l}\text { Stop Maintenance } \\
T^{*}\end{array}$ & $\begin{array}{c}\text { Sale Date } \\
T\end{array}$ & & & & \\
\hline 1 & 0.0 & 28.6 & 47.2 & 502.3 & $m^{*}=$ & 2 & $0 \leqslant t \leqslant 47.2$ \\
\hline 2 & 0.005 & 27.5 & 45.3 & 501.0 & $m^{*}=$ & $\left\{\begin{array}{l}1 \\
2\end{array}\right.$ & $\begin{array}{c}0 \leqslant t \leqslant 0.3 \\
0.3<t \leqslant 45.3\end{array}$ \\
\hline 3 & 0.01 & 27.2 & 45.3 & 500.9 & $m^{*}=$ & $\left\{\begin{array}{l}1 \\
2 \\
1\end{array}\right.$ & $\begin{array}{c}0 \leqslant t \leqslant 0.3 \\
0.3<t \leqslant 44.1 \\
44.1<t \leqslant 45.3\end{array}$ \\
\hline 4 & 0.02 & 27.2 & 45.3 & 500.5 & $m^{*}=$ & $\begin{array}{l}1, \\
2, \\
1,\end{array}$ & $\begin{array}{c}0 \leqslant t \leqslant 0.9 \\
0.9<t \leqslant 44.1 \\
44.1<t \leqslant 45.3\end{array}$ \\
\hline 5 & 0.03 & 27.1 & 45.2 & 500.1 & $m^{*}=\{$ & $\begin{array}{l}1, \\
2, \\
1, \\
2,\end{array}$ & $\begin{array}{c}0 \leqslant t \leqslant 1.2 \\
1.2<t \leqslant 16.0 \\
16.0<t \leqslant 17.2 \\
17.2<t \leqslant 45.2\end{array}$ \\
\hline 6 & 0.05 & 27.1 & 45.2 & 499.2 & $m^{*}=$ & 1 , & $0 \leqslant t \leqslant 45.2$ \\
\hline 7 & 0.1 & 27.1 & 45.1 & 498.3 & $m^{*}=$ & 1, & $0 \leqslant t \leqslant 45.1$ \\
\hline 8 & 0.2 & 27.0 & 45.1 & 496.4 & $m^{*}=$ & 1 , & $0 \leqslant t \leqslant 45.1$ \\
\hline 9 & 0.4 & 26.7 & 44.5 & 492.5 & $m^{*}=$ & 1, & $0 \leqslant t \leqslant 44.5$ \\
\hline 10 & 1.0 & 26.2 & 43.3 & 480.9 & $m^{*}=$ & 1, & $0 \leqslant t \leqslant 43.3$ \\
\hline
\end{tabular}

where $\sum_{j=0}^{N} q_{i j}^{m u}(t)=0, i=0,1, \cdots, N$. Superscripts $m$ and $u$ denote dependence of the elements of $Q$ on the number of repairmen and PM. In the process control situation, an LQG formulation is appropriate and the objective is to choose an optimal feedback control $v^{*}(t)$ to minimize a performance index of the form

$$
E\{J\}=E\left\{\int_{0}^{T}\left[x^{\prime}(t) R_{1} x(t)+v^{\prime}(t) R_{2} v(t)\right] d t\right\} .
$$

The advantages of this performance index are well known in process control. Combined optimal strategies $u^{*}, m^{*}$, and $v^{*}$ for the integrated system may be obtained by optimizing an overall index of the form $f(J, V)$. But to make the problem more tractable, the problems may be solved sequentially with the solution of the economic model being an input to the process control model. The optimal PM policy $u^{*}(t)$ and $m^{*}(t)$ are obtained by the methods of Sections II and III. $u^{*}$ and $m^{*}$ determine the matrix $Q^{m u}(t)$ of (8). An optimal feedback controller $v^{*}(x(t), t)$ for the process control system may be obtained by minimizing (9) subject to (6) and is given by [9]

$$
v^{*}(t, x(t), j)=-B_{j}^{*} K_{j}(t) x(t)
$$

where $K_{j}(t)$ are the solutions of

$d K_{j}(t) / d t=-A_{j}^{\prime} K_{j}(t)-K_{j}(t) A_{j}(t)-K_{j}(t) B_{j} B_{j}^{\prime} K_{j}(t)$

$$
-\sum_{i=0}^{N} K_{i}(t) q_{j i}^{m^{*} u^{*}}(t)+R
$$

$$
K_{j}(T)=0, \quad j=0,1, \cdots, N
$$

where integers $j$ denote the operational modes of the system, i.e., the number of machines not working at any time $t$.

\section{CONCluding Remarks}

This short paper presents an approach for obtaining the optimal preventive maintenance policies and optimal repairmen policies for machines subject to deterioration, intermittent breakdowns, and repairs. The waiting line formed at the service facility is considered and the usual steady-state assumption on the queue is relaxed by directly considering the Chapman-Kolmogorov equations as state equations of the system. It may be observed that we obtain open-loop optimal policies for the economic decision model. On the other hand, when the system of machines is part of a process control system the effect of breakdowns and repairs on feedback control laws is examined. Results show that the optimal process control law involves feedback of process states and information feedback on the operational status of the machines. It may, however, be noted that detailed replacement analysis is not considered in this short paper. If a longer planning horizon is considered, technological innovations of challenging machines are to be considered together with the obsolescence of previous machines and these will have some effect on PM policies.

\section{ACKNOWLEDGMENT}

The authors wish to thank the reviewers for several valuable suggestions. They also wish to thank Dr. N. Viswanadham for useful discussions. 


\section{REFERENCES}

[1] J. J. McCall, "Maintenance policies for stochastically failing systems: A survey," Management Sci.. vol. 11, pp. 493-525, Mar. 1965.

[2] B. Naslund, "Simultaneous determination of optimal repair policy and service life," Swed. J. Econ., vol. LXVIII, no. 2, pp. 63-73, 1966.

[3] G. L. Thompson, "Optimal maintenance policy and sale date of a machine," Management Sci., vol. 14. pp. 643-650. May 1968.

[4] S. R. Arora and P. T. Lele, "A note on optimal maintenance policy and sale date of a machine," Management Sci., vol. 17, pp. 170-173, Nov. 1970.

[5] M. I. Kamien and N. L. Schwartz, "Optimal maintenance and sale age for a machine subject to failure," Management Sci.. vol. 17, pp. 495-504, Apr. 1971.

[6] M. Alam and V. V. S. Sarma. "Optimum maintenance policy for an equipment subject to deterioration and random fajlure," IEEE Trans. Syst., Man, Cybern., vol. SMC-4, pp. 172-175, Mar. 1974

[7] S. P. Sethi, "Simultaneous optimization of preventive maintenance and Replacement policy for machines; A modern control theory approach," AIIE Trans., vol. 5 . pp. 156-163. June 1973 .

[8] V. V. S. Sarma and M. Alam, "Optimal maintenance policies for machines subject to deterioration and intermittent breakdowns," IEEE Trans. Syst., Man, Cybern. (Corresp.), vol. SMC-5, pp. 396-398. May 1975.

[9] D. D. Sworder and T. Kazangey. "Optimal control, repair, and inventory strategies for a linear stochastic system," IEEE Trans. Syst., Man, Cybern., vol. SMC-2, pp. 342-347, July 1972.

[10] D. G. Luenberger, "A nonlinear economic control problem with a linear feedback solution," IEEE Trans. Akitomat. Contr., vol. AC-20, pp 184-191, Apr. 1975.

[11] W. Feller, An Introduction to Probability Theory and Its Applications, vol. 1. New York: Wiley, 1967.

[12] A. E. Bryson and Y. C. Ho, Applied Optimal Control. Waltham, MA: Blaisdell. 1969.

[13] B. Pagurek and M. Woodside, "The conjugate gradient method for optimal control problems with bounded control variables," Automatica, vol. 4, pp. 337-349, Nov. 1968.

[14] M. Alam, “Optimal preventive maintenance, replacement and repairmen strategies for stochastically failing systems: A modern control theory approach," Ph.D. dissertation, Indian Inst. Sci., Bangalore, India, Aug. 1974.

\section{On the Exact Model Matching Controller Design}

\section{S. G. TZAFESTAS, MEMBER, IEEE, AND P. N. PARASKEVOPOULOS}

Abstract-The problem of designing a static state feedback controller which matches a given multivariable system to a desired ideal system (model) is treated. The system under control is assumed in state space form while the model is assumed in transfer matrix form. An algorithm is developed which separates the conditions which must be satisfied by the system under control and the model to be matched from the equations which must be solved to find the gains of the feedback controller. Two examples are included.

\section{INTRODLCTION}

The model-matching problem by using feedback (static or dynamic) is of primary importance in the automatic control field and involves pole assignment, zero assignment, the input-output decoupling problem, etc., as special cases. Most of the available results [1]-[4] concern the case of static feedback, and only in [3]. [4] the dynamic feedback case is studied. The mathematical tools used are algebraic due to the nature of the exact model-matching (or model-following) problem although some authors have treated the problem from an optimal regulator point of view [5]. [6].

In the present short paper we present a new approach in which the system under control is in the state space representation form while the model is in transfer matrix function form. The system under control is treated directly in the time domain and converted in the input-output form. Comparing the input-output form of the system to that of the given model gives a set of equations the solution of which provides the feedback law required.

Manuscript received April 8, 1974; revised December 2, 1975. Paper recommended by D. G. Lainiotis. Past Chairman of the IEEE S-CS Adaptive and Learning Systems, Pattern Recognition Committee. This paper is a concise edition of a paper presented at the 1974 IEEE Conference on Decision and Control

S. G. Tzafestas is with the Department of Electrical Engineering, University of Patras, Patras, Greece and the Department of Reactors, N.R.C.. "Demokritos," Athens, Greece.

P. N. Paraskevopoulos is with the Computer Control Section. Computer Center, N.R.C., "Demokritos," Aghia Paraskevi Attikis, Athens, Greece.
To solve these equations an iterative algorithm is utilized which essentially splits them into two sets, the first set does not depend on the feedback gains sought but only upon the system under control-model pair, and the second depends both on the system-model pair and the feedback gains $F$ and $G$. The first set is actually the set of exact model-matching conditions while the second one is the set of the exact model-matching design equations.

The set of exact model-matching design equations is nonlinear in $F$ and $G$ and the general solution so far has not been derived. Here, we present solutions in three particular cases which, however, possess a great degree of generality, involve all known results as special cases and cover new situations not treated in the past. Finally, the model-matching problem by output feedback is treated and two examples are given which illustrate the applicability of the theory.

\section{Formul.ation of the Problem}

Consider a linear time-invariant system in state-space of the type

$$
\dot{x}=A x+B u, \quad y=C x+D u
$$

where $x(t) \in R_{n}, u(t) \in R_{m}, y(t) \in R_{p}$, and $A, B, C$, and $D$ given constant matrices of appropriate sizes. Now, consider a dynamic model with given transfer matrix function of the type

$$
T_{m}(s)=\frac{1}{\varphi(s)} \sum_{k=0}^{n} M_{k} s^{k}, \quad \varphi(s)=\sum_{n=0}^{n} a_{k} s^{k}, \quad a_{n}=1
$$

where $M_{k}$ are $p \times r$ constant matrices and $\varphi(s)$ is the characteristic polynomial of the model.

The problem of exact model-matching can be stated as follows. Given a dynamic system of the form (1) determine a linear state feedback law $u=F x+G \omega$ such that the transfer function (input-output description) of the closed-loop system

$$
\dot{x}=(A+B F) x+B G \omega, \quad y=(C+D F) x+D G \omega
$$

is the same as that of a given model.

\section{Solltion of the Problem}

The transfer function of the closed-loop system (3) is $T_{c}(s)=(C+D F)$ $(s I-A-B F)^{-1} B G+D G$; and hence for exact model-matching the pair $\{F, G\}$ must be selected such that

$$
T_{c}(s)=T_{m}(s) .
$$

Equation (4) is the first form (the "crudest" form) of the relation which must be satisfied for exact model-matching by state feedback. Several investigators $[1\} 2]$ have based their work directly on (4) and tried to determine $\{F, G\}$ by using and working on the above form of $T_{c}(s)$. Here we shall adopt a kind of reverse procedure, namely, that of computing $T_{c}(s)$ utilizing the description (1) directly in the time domain. This procedure of calculating $T_{c}(s)$ is as follows.

1) Obtain the time derivatives up to the $n$th one of the output vector $y$ in the closed-loop system (3).

2) Utilize the Cayley-Hamilton theorem

$$
(A+B F)^{n}=\sum_{k=0}^{n-1} p_{k}(F)(A+B F)^{k}
$$

where $p_{k}(F)$ are scalars depending on $A+B F$ (since $A$ and $B$ are fixed, these scalars actually depend only on $F$ ), in the result of 1) to eliminate $x$.

3) Take the Laplace transform of the equation resulted in 2) with zero initial conditions.

Equating the resulting transfer function $T_{c}(s)$ in 3 ) with the modeltransfer function $T_{m}(s)$ and after some algebraic manipulation we get the following set of equations:

$$
\begin{aligned}
D G & =H_{n} \\
(C+D F)(A+B F)^{k} B G & =H_{n-k-1}, \quad k=0,1, \cdots, n-1
\end{aligned}
$$

